\title{
Iron Replacement Therapy with Oral Ferric Maltol: Review of the Evidence and Expert Opinion
}

\author{
Carsten Schmidt ${ }^{1,2, *}$, Stephen Allen ${ }^{3}\left(\mathbb{D}\right.$, Nelson Kopyt $^{4}$ and Pablo Pergola ${ }^{5}$ \\ 1 Medical Clinic II, Department of Gastroenterology, Hepatology, Endocrinology, Diabetology and Infectious \\ Diseases, Klinikum Fulda, Pacelliallee 4, 36043 Fulda, Germany \\ 2 Medical Faculty, Friedrich Schiller University, 07747 Jena, Germany \\ 3 Department of Clinical Sciences, Liverpool School of Tropical Medicine, Pembroke Place, \\ Liverpool L3 5QA, UK; stephen.allen@lstmed.ac.uk \\ 4 Department of Medicine, Division of Nephrology, Lehigh Valley Hospital, 1230 S Cedar Crest Boulevard, \\ Suite 301, Allentown, PA 18103, USA; nkopyt@gmail.com \\ 5 Renal Associates PA, 1123 N Main Av., Suite 120, San Antonio, TX 78212, USA; ppergola@raparesearch.com \\ * Correspondence: carsten.schmidt@klinikum-fulda.de; Tel.: +49-(0)661-845421
}

Citation: Schmidt, C.; Allen, S.; Kopyt, N.; Pergola, P. Iron

Replacement Therapy with Oral Ferric Maltol: Review of the Evidence and Expert Opinion. J. Clin. Med. 2021, 10, 4448. https://doi.org/ $10.3390 /$ jcm 10194448

Academic Editor: Giacomo Garibotto

Received: 17 August 2021

Accepted: 23 September 2021

Published: 28 September 2021

Publisher's Note: MDPI stays neutral with regard to jurisdictional claims in published maps and institutional affiliations.

Copyright: (c) 2021 by the authors. Licensee MDPI, Basel, Switzerland. This article is an open access article distributed under the terms and conditions of the Creative Commons Attribution (CC BY) license (https:// creativecommons.org/licenses/by/ $4.0 /)$.

\begin{abstract}
Iron deficiency is the most common cause of anemia globally and is frequently reported in patients with underlying inflammatory conditions, such as inflammatory bowel disease (IBD) and chronic kidney disease (CKD). Ferric maltol is a new oral iron replacement therapy designed to optimize iron absorption while reducing the gastrointestinal adverse events associated with unabsorbed free iron. Ferric maltol has been studied in clinical trials involving almost 750 adults and adolescents with iron-deficiency anemia associated with IBD, CKD, and other underlying conditions, and it has been widely used in clinical practice. It is approved for the treatment of adults with iron deficiency with or without anemia, independent of the underlying condition, and is commercially available in Europe and the United States. We review the published evidence for ferric maltol, which demonstrates consistent and clinically meaningful improvements in hemoglobin and measures of iron availability (ferritin and transferrin saturation) and shows that it is well-tolerated over longterm treatment for up to 64 weeks-an important consideration in patients with chronic underlying conditions such as IBD and CKD. We believe that ferric maltol is an effective, convenient, and well-tolerated treatment option for iron deficiency and iron-deficiency anemia, especially when long-term management of chronic iron deficiency is required. Writing support was provided by Shield Therapeutics (Gateshead, UK).
\end{abstract}

Keywords: adult; anemia; chronic kidney disease; ferric maltol; hemoglobin; inflammatory bowel disease; iron deficiency; pediatric; pulmonary hypertension; tolerability

\section{Introduction}

Iron deficiency refers to any situation in which there is insufficient iron available in the body to meet physiologic needs. It is the most common nutritional disorder in humans, accounting for around $50 \%$ of the estimated 2.2 billion cases of anemia (low hemoglobin) worldwide [1-4]. Absolute iron deficiency can develop as a result of malnutrition, malabsorption, or blood loss. Inflammatory bowel disease (IBD) is further complicated by absolute iron deficiency due to chronic gastrointestinal blood loss and impaired iron absorption across the damaged bowel mucosa, as well as inflammation-associated (hepcidinmediated) downregulation of iron absorption [5]. In addition, inflammatory disorders such as IBD and chronic kidney disease (CKD) may result in functional iron deficiency, with impaired iron absorption and release from storage proteins resulting in inappropriate iron availability to meet metabolic demands, such as erythropoiesis $[4,6]$.

Iron deficiency adversely affects overall health and well-being, even before the development of anemia [7-12]. Iron deficiency, with or without anemia, is associated with fatigue, 
impaired physical and cognitive function, headache, tachycardia, and dyspnea [7-17], with resulting adverse impacts on daily activities, productivity, quality of life, and any additional underlying illnesses [7-15,18]. The wide-ranging effects of iron deficiency reflect the crucial role that iron plays in oxygen transport, enzymatic reactions, cellular processes, immunity, and cognitive function $[4,19,20]$.

Iron absorption, transport, storage, and use are normally tightly regulated, with daily small losses in the urine, feces, or sweat replaced by iron in the diet [6]. Once absorbed, most iron is incorporated into hemoglobin in erythrocytes, while some is converted to myoglobin in muscle tissue. Iron that is not immediately needed can be stored in ferritin or hemosiderin [21-23]. The transport of iron from cell to cell is achieved via iron transporter mechanisms, including ferroportin, which mediates iron transport out of the cell from the cytoplasm across the cell membrane; transferrin, which binds iron in the plasma for delivery to tissues; and transferrin receptors on cell surfaces, which allow the iron to be internalized $[6,21,23]$. Ferroportin activity is regulated by hepcidin in response to the body's iron requirements: in the presence of high iron levels, hepcidin is upregulated, binding to ferroportin to stimulate degradation of the transport protein, thereby preventing the movement of iron out of the cell and reducing the amount of available iron [6,22,23]. Hepcidin production is also stimulated by cytokines, resulting in the low availability of iron in inflammatory conditions [21-23]. In patients with kidney dysfunction, such as CKD without dialysis, renal clearance of hepcidin is impaired, thus also resulting in the accumulation of hepcidin and contributing to the ongoing disruption of iron absorption and recycling [22].

Low hemoglobin levels $(<13 \mathrm{~g} / \mathrm{dL}$ in men, $<12 \mathrm{~g} / \mathrm{dL}$ in nonpregnant women and children aged $>11$ years, $<11 \mathrm{~g} / \mathrm{dL}$ in pregnant women and younger children [1]) may indicate insufficient iron available for erythropoiesis (i.e., anemia). In addition, the molecules involved in iron regulation are used as markers of iron availability and iron deficiency. Ferritin is upregulated in the presence of iron [21]; thus, high concentrations may indicate high iron availability, whereas low concentrations (e.g., $<15 \mu \mathrm{g} / \mathrm{L}$ in adults) reliably indicate iron deficiency $[4,6]$. As ferritin is upregulated in inflammatory conditions such as IBD or CKD [21], a higher cut-off may be required (e.g., $<30$ or $<100 \mu \mathrm{g} / \mathrm{L}$ ) to define iron deficiency $[4,6,24]$. Transferrin molecules have two iron-binding sites, but typically the average proportion of overall binding sites occupied (termed transferrin saturation (TSAT)) is $30-50 \%$; a TSAT of $\leq 20 \%$ indicates low iron availability and can be used to diagnose iron deficiency, particularly in the presence of low ferritin levels $[4,19,21]$.

\section{Iron Replacement Therapy}

In patients with iron-deficiency anemia, and in many of those with iron deficiency alone, iron replacement therapy is required in order to support physiologic processes and maintain quality of life, cognitive functioning, and the ability to complete daily activities. It has been estimated that $500 \mathrm{mg}$ of absorbed iron are needed to raise hemoglobin levels by $2 \mathrm{~g} / \mathrm{dL}$, which is generally accepted as a meaningful increase in patients with anemia [24]; this amount can be delivered as a single intravenous (IV) iron infusion or as daily oral iron taken for $\geq 4$ weeks $[25,26]$. However, although this amount of iron addresses the immediate needs of patients with anemia, it may not replenish the body's iron stores sufficiently to overcome chronic iron deficiency. Particularly in patients with underlying inflammatory diseases, longer-term iron replacement therapy is required to raise and maintain iron stores physiologically, alongside the correction or control of the underlying condition to minimize further loss of iron $[5,26]$.

Oral iron replacement therapy, usually in the form of ferrous salts, offers convenience in terms of low cost and easy administration [27]. However, the effectiveness of many oral formulations may be reduced by limited bioavailability, particularly in patients with underlying inflammatory conditions, because only 10-20\% of iron from oral ferrous formulations is estimated to be absorbed $[25,28]$. Unabsorbed iron can form reactive hydroxyl radicals in the gut [26,29-32], leading to mucosal irritation or damage [26,29,32-36]. The resulting 
gastrointestinal adverse events, such as nausea, epigastric discomfort, and constipation, may reduce patients' willingness to continue treatment $[25,26,28,30,35,36]$. Unabsorbed iron may also affect the gut microbiome [26,30,32,36-39] and can trigger disease flares in patients with IBD $[26,31,33,36,37]$. Absorption may be improved if treatment is taken with ascorbic acid or on an empty stomach to increase or maintain gastrointestinal acidity $[26,36]$. Tolerability may be improved with concurrent food intake, lower doses, or longer intervals between dosing, although the correction of iron deficiency may be slower as a result $[25,26,28,36]$. The World Health Organization recommends a maximum oral dosage of elemental iron of $60 \mathrm{mg}$ per day [40], equivalent to approximately $325 \mathrm{mg}$ of ferrous sulfate.

Intravenous iron offers rapid iron replacement by bypassing endogenous iron uptake mechanisms in the gut and is thus useful in patients with significant iron depletion requiring rapid replacement [41]. In terms of immediate iron replacement, IV iron is more effective than oral iron, but there is limited evidence on the long-term impact of IV versus oral iron on healthcare resource use, adverse events, and patients' quality of life [42]. When using IV iron, physicians must consider the higher cost and resource requirements compared with oral irons. Administration of IV iron may require a hospital or clinic setting, and there are small but potentially serious risks of anaphylaxis, hypophosphatemia, and iron overload (particularly in the presence of functional iron deficiency, when effective processing of available iron is disrupted) $[36,43,44]$.

An easy-to-use oral iron therapy with good absorption and low risk of gastrointestinal adverse events would minimize the burden of treatment on patients requiring long-term iron replacement. Various alternatives to oral ferrous iron formulations have been developed, including polysaccharide-ferric iron complexes, sucrosomial iron, sodium feredate, ferric citrate, and ferric maltol [27,45-47].

\section{Ferric Maltol}

Ferric maltol was rationally designed to optimize the absorption and tolerability of oral iron [48]. It has been approved by the European Medicines Agency, SwissMedic, and the US Food and Drug Administration and is commercially available in Europe and the United States for the treatment of adults with iron deficiency, with or without anemia. Regulatory approval was based on a comprehensive program of research, including clinical trials in a variety of settings in adults (IBD, CKD, and pulmonary hypertension). The absorption of iron from ferric maltol has also been investigated in adolescents (aged 10-17 years). To review the evidence base for ferric maltol, we searched PubMed and Google Scholar for articles, excluding patents, published up to 31 May 2021, using the free text search strings (ferric maltol) and (ferric trimaltol), with no date or language restrictions. We supplemented the search results with our own articles in development (now peer-reviewed and published) and congress presentations identified by the manufacturer (Shield Therapeutics (UK) Ltd., Gateshead, UK). We identified 34 publications reporting data on ferric maltol (Supplementary Figure S1 in the Supplementary Materials), including 12 reports of preclinical and clinical pharmacology research [49-60], 13 reports of clinical efficacy/effectiveness and safety [61-73], and 9 reports of health economic or patientreported outcomes (not reviewed further here) [74-82]. While drafting this article, we identified two additional publications, which we have also summarized below $[83,84]$.

\subsection{Preclinical and Pharmacology Studies}

\subsubsection{Preclinical Evidence}

Ferric maltol is a complex of ferric iron and maltol (3-hydroxy-2-methyl-4-pyrone), a naturally occurring sugar derivative found in many food products, which is highly selective for iron [49]. The iron-maltol complex is stable at a physiologic $\mathrm{pH}$ [55] and, as shown in vitro and in vivo, remains strongly chelated until the point of absorption in the gut, when the greater affinity of iron for the iron transport receptor promotes dissociation [50,51,54]. Once dissociated, as demonstrated in vitro, ferric iron is readily transported across the 
gut lumen [51]. Maltol is separately absorbed, metabolized, and rapidly eliminated in the urine [54].

Studies of ferric maltol in rats have demonstrated that the dissociated iron is absorbed even in models of intestinal damage, with a plateauing effect at higher doses in both healthy and damaged guts, indicating saturable absorption $[49,53]$. More effective iron absorption from the iron-maltol complex was recorded when iron was in the ferric versus ferrous form [49].

Unabsorbed iron remains chelated in the iron-maltol complex until excretion in the feces, thereby potentially reducing the risk of intestinal damage from free iron [53]. Mice with chemically induced colitis had no change in fecal iron content or colitis features after 10 days of treatment with ferric maltol, whereas fecal iron increased significantly, and features of colitis worsened significantly in mice treated with ferrous sulfate [83]. Other researchers have cited the presence of fecal iron as a likely factor in the adverse effects of oral ferrous sulfate therapy in humans $[29,85,86]$, so these preclinical data in mice indicate a potential mechanism for reduced gastrointestinal toxicity with ferric maltol in clinical use. Furthermore, ferric maltol supplementation was associated with fewer changes in the murine microbiome compared with ferrous sulfate. Fecal samples from humans with iron deficiency also showed no significant change in the microbiome from baseline (pretreatment) following treatment with ferric maltol, whereas many genera changed significantly following treatment with ferrous sulfate [83]. These data support a potentially protective effect of ferric maltol on the gut microbiome and an avoidance of free iron-induced intestinal damage.

\subsubsection{Clinical Pharmacology}

In human studies, iron from ferric maltol was at least as well absorbed as ferrous iron, and it was better absorbed from the complex than from simple ferric salts [55,56]. Iron absorption was increased five-fold when ferric maltol was taken on an empty stomach versus with food [87]. Ferric maltol showed predictable pharmacokinetics with repeated dosing in adults and adolescents (aged 10-17 years) [59,73]. Uptake was rapid, with maximum concentrations $\left(C_{\max }\right)$ of iron in the plasma achieved within $2-3 \mathrm{~h}$ after administration in adults with IBD [59]. In iron-deficient adults, serum iron levels increased substantially following oral administration of ferric maltol, whereas considerably less absorption was seen in iron-replete adults [56,57], confirming effective absorption of iron with physiologic control of the uptake to meet the body's needs. Mean ferritin concentrations increased over time, indicating the replenishment of iron stores [59].

In adults with IBD, maltol and maltol glucuronide concentrations increased rapidly and dose proportionally in the plasma $\left(C_{\max } 1-1.5 \mathrm{~h}\right.$ post dose); the ligand was rapidly excreted as maltol glucuronide in the urine within $3 \mathrm{~h}$, and no accumulation was seen with repeated dosing [59]. Similarly, in adolescents (aged 10-17 years), maltol concentrations increased rapidly and dose dependently, and maltol was completely metabolized to maltol glucuronide $2-3 \mathrm{~h}$ after administration of the iron-maltol complex [73].

\subsubsection{Posology}

In Europe and the United States, the approved adult dosage of ferric maltol is $30 \mathrm{mg}$ twice daily (total $60 \mathrm{mg}$ elemental iron/day), to be taken on an empty stomach to maximize absorption. Treatment duration is dependent on the severity of iron deficiency, but generally at least 12 weeks of treatment is required. It is recommended that treatment is continued as long as necessary to replenish the body's iron stores according to blood tests [88-90].

\subsection{Clinical Evidence Base}

\subsubsection{Study Designs}

Ferric maltol has been studied in phase I-III clinical trials involving 624 adults and 37 adolescents (aged 10-17 years) with iron deficiency [59,61,62,64,68,71-73]. Full details 
of these studies, as well as two reports of real-world experience in 87 adults [69,70], are provided in Supplementary Table S1 in the Supplementary Materials. Four phase III or IIIB trials in adults (two in patients with quiescent or mild to moderate IBD, one in patients with non-dialysis-dependent stage III or IV CKD, and one in patients with pulmonary hypertension) and a phase I pediatric (adolescent) trial are described here (Table 1) $[62,64,68,71-73]$. In the adult trials, ferric maltol was given at the approved dosage of $30 \mathrm{mg}$ twice daily for $\geq 12$ weeks ( $\geq 16$ weeks in the CKD study) $[62,68,71,72]$. In the pediatric study, patients were randomized to receive ferric maltol 7.8, 16.6, or $30 \mathrm{mg}$ twice daily for 10 days [73].

Table 1. Designs of key ferric maltol clinical studies.

\begin{tabular}{|c|c|c|c|c|c|c|c|}
\hline Study & Location & Underlying Condition & $\begin{array}{l}\text { Anemia and } \\
\text { Iron Deficiency } \\
\text { Definitions }\end{array}$ & Patients, N & Design & Comparator & $\begin{array}{l}\text { Primary } \\
\text { Endpoint }\end{array}$ \\
\hline \multicolumn{8}{|l|}{ Adult } \\
\hline $\begin{array}{c}\text { Phase III IBD } \\
(\text { AEGIS 1/2) } \\
{[62,64]}\end{array}$ & Global & $\begin{array}{c}\text { Eligibility criteria: } \\
\text { Quiescent or mild or moderate } \\
\text { IBD } \\
\text { UC: SCCAI score }<4 \text { at } \\
\text { screening and randomization } \\
\text { CD: CDAI score }<220 \text { at } \\
\text { randomization } \\
\text { At baseline: } \\
\text { UC: FM } n=29 \text {; placebo } n=29 \\
\text { Median (range) SCCAI score: } \\
\text { FM } 2.0(0-3) \text {; placebo } 2.0(0-3) \\
\text { CD: FM } n=35 ; \text { placebo } n=35 \\
\text { Median (range) CDAI score: } \\
\text { FM } 75 \text { (14-199); placebo } 108 \\
\text { (10-220) }\end{array}$ & $\begin{array}{c}\mathrm{Hb} \geq 9.5 \text { to } \\
<12.0 \mathrm{~g} / \mathrm{dL} \\
\text { (women) or } \\
<13.0 \mathrm{~g} / \mathrm{dL}(\mathrm{men}) \\
\text { Ferritin } \\
<30 \mu \mathrm{g} / \mathrm{L} \text { at } \\
\text { screening }\end{array}$ & $\begin{array}{c}128(\mathrm{FM} n=64 ; \\
\text { placebo } n=64) \\
97 \text { started OL } \\
\text { FM after DB } \\
\text { FM }(n=50) \text { or } \\
\text { DB placebo } \\
(n=47)\end{array}$ & $\begin{array}{l}\text { Randomized, } \\
\text { DB, } \\
\text { superiority52- } \\
\text { week OL } \\
\text { extension }\end{array}$ & $\begin{array}{l}\text { Placebo (DB } \\
\text { period only) }\end{array}$ & $\begin{array}{l}\mathrm{Hb} \text { change } \\
\text { from baseline } \\
\text { to week } 12\end{array}$ \\
\hline $\begin{array}{c}\text { Phase IIIB IBD } \\
(\mathrm{H} 2 \mathrm{H})[71]\end{array}$ & Global & $\begin{array}{c}\text { Eligibility criteria: } \\
\text { Quiescent or mild or moderate } \\
\text { IBD } \\
\text { UC: SCCAI score } \leq 5 \text { during } \\
\text { screening } \\
\text { CD: CDAI score } \leq 300 \text { during } \\
\text { screening } \\
\text { At baseline: } \\
\text { UC: FM } n=46 \text {, IV FCM } n=46 \\
\text { Mean (SD) SCCAI score: FM } \\
2.2(1.8) \text {; IV FCM } 2.3(1.6) \\
\text { CD: FM } n=79, \text { IV FCM } n=79 \\
\text { Mean (SD) CDAI score: FM } \\
\text { 129.6 (60.1); IV FCM 140.5 } \\
\text { (75.8) }\end{array}$ & $\begin{array}{c}\mathrm{Hb} \geq 8.0 \text { to } \\
\leq 11.0 \mathrm{~g} / \mathrm{dL} \\
\text { (women) or } \\
\leq 12.0 \mathrm{~g} / \mathrm{dL} \\
\quad \text { (men) } \\
\text { Ferritin } \\
<30 \mu \mathrm{g} / \mathrm{L} \text { or } \\
\text { ferritin } \\
<100 \mu \mathrm{g} / \mathrm{L}+ \\
\text { TSAT }<20 \%\end{array}$ & $\begin{array}{c}250 \text { (ITT: FM } \\
n=125, \text { IV } \\
\text { FCM } n=125 ; \\
\text { PP: FM } n=78 \\
\text { IV FCM } \\
n=88 \text { ) }\end{array}$ & $\begin{array}{c}\text { Randomized, } \\
\text { OL, } \\
\text { non-inferiority }\end{array}$ & IV FCM & $\begin{array}{l}\text { Hb responder } \\
\text { rate at week } 12 \\
(\geq 2 \mathrm{~g} / \mathrm{dL} \\
\text { increase or } \\
\text { normalization })\end{array}$ \\
\hline $\begin{array}{c}\text { Phase III CKD } \\
\text { [72] }\end{array}$ & USA & $\begin{array}{c}\text { Eligibility criteria: } \\
\text { CKD stage III or IV (eGFR } \geq 15 \\
\text { to }<60 \mathrm{~mL} / \mathrm{min} / 1.73 \mathrm{~m}^{2}, \text { not } \\
\text { on dialysis) } \\
\text { At baseline:Mean (SD) eGFR: } \\
\text { FM } 31.9(11.5) \\
\mathrm{mL} / \mathrm{min} / 1.73 \mathrm{~m}^{2} \\
\mathrm{Placebo} 29.7(10.6) \\
\mathrm{mL} / \mathrm{min} / 1.73 \mathrm{~m}^{2}\end{array}$ & $\begin{array}{l}\mathrm{Hb} \geq 8.0 \text { to } \\
<11.0 \mathrm{~g} / \mathrm{dL} \\
\text { Ferritin } \\
<250 \mu \mathrm{g} / \mathrm{L}+ \\
\text { TSAT }<25 \% \text { or } \\
\quad \text { ferritin } \\
<500 \mu \mathrm{g} / \mathrm{L}+ \\
\text { TSAT }<15 \%\end{array}$ & $\begin{array}{c}167(\mathrm{FM} \\
n=111 ; \\
\text { placebo } n=56) \\
125 \text { started OL } \\
\text { FM after } 16 \\
\text { weeks of DB } \\
\text { FM }(n=86) \text { or } \\
\text { DB placebo } \\
(n=39) \\
\end{array}$ & $\begin{array}{l}\text { Randomized, } \\
\text { DB, } \\
\text { superiority36- } \\
\text { week OL } \\
\text { extension }\end{array}$ & Placebo & $\begin{array}{l}\text { Hb change } \\
\text { from baseline } \\
\text { to week } 16\end{array}$ \\
\hline $\begin{array}{l}\text { Phase IIIB PH } \\
{[68]^{\prime}}\end{array}$ & Germany & $\begin{array}{c}\text { Eligibility criteria: } \\
\text { any form of PH with mean } \\
\text { resting pulmonary artery } \\
\text { pressure } \geq 25 \text { mmHg } \\
\text { At baseline: } \\
\text { PAH }(n=14) \\
\text { PH due to } \\
\text { left heart disease }(n=1) \\
\text { Inoperable chronic } \\
\text { thromboembolic PH }(n=7) \\
\text { Mean (SD) pulmonary artery } \\
\text { pressure } 50(11) \text { mmHg }\end{array}$ & $\begin{array}{c}\mathrm{Hb} \geq 7 \text { to } \\
<12 \mathrm{~g} / \mathrm{dL} \\
\text { (women) or } \geq 8 \\
\text { to }<13 \mathrm{~g} / \mathrm{dL} \\
\text { (men) } \\
\text { Ferritin } \\
<100 \mu \mathrm{g} / \mathrm{L} \text { or } \\
\text { ferritin } \\
100-300 \mu \mathrm{g} / \mathrm{L}+ \\
\text { TSAT }<20 \%\end{array}$ & 22 & $\begin{array}{l}\text { Single-arm OL, } \\
\text { exploratory }\end{array}$ & None & $\begin{array}{l}\text { Hb change } \\
\text { from baseline } \\
\text { to week } 12\end{array}$ \\
\hline
\end{tabular}


Table 1. Cont.

\begin{tabular}{|c|c|c|c|c|c|c|c|}
\hline Study & Location & Underlying Condition & $\begin{array}{l}\text { Anemia and } \\
\text { Iron Deficiency } \\
\text { Definitions }\end{array}$ & Patients, N & Design & Comparator & $\begin{array}{c}\text { Primary } \\
\text { Endpoint }\end{array}$ \\
\hline \multicolumn{8}{|l|}{ Pediatric } \\
\hline Phase I [73] & UK & $\begin{array}{c}\text { Eligibility criteria: } \\
\text { Iron deficiency of any cause } \\
\text { At baseline: } \\
\text { CD }(n=8) \\
\text { Other gastrointestinal } \\
\text { disorders }(n=11) \\
\text { Vitamin D deficiency }(n=7) \\
\text { CKD }(n=4) \\
\text { Other conditions }(n=7)\end{array}$ & $\begin{array}{c}\text { Ferritin } \\
<30 \mu \mathrm{g} / \mathrm{L} \text { or } \\
\text { ferritin }<50 \mu \mathrm{g} / \mathrm{L} \\
+ \text { TSAT }<20 \%\end{array}$ & 37 & $\begin{array}{c}\text { Randomized, } \\
\text { exploratory }\end{array}$ & $\begin{array}{l}\text { Different FM } \\
\text { doses }\end{array}$ & $\begin{array}{c}\text { PK, iron } \\
\text { uptake }\end{array}$ \\
\hline
\end{tabular}

CD, Crohn's disease; CDAI, Crohn's Disease Activity Index; CKD, chronic kidney disease; DB, double-blind; eGFR, estimated glomerular filtration rate; FCM, ferric carboxymaltose; FM, ferric maltol; $\mathrm{H} 2 \mathrm{H}$, head-to-head; $\mathrm{Hb}$, hemoglobin; IBD, inflammatory bowel disease; ITT, intention-to-treat; IV, intravenous; OL, open-label; PAH, pulmonary arterial hypertension; PH, pulmonary hypertension; PK, pharmacokinetics; PP, per protocol; SCCAI, Simple Clinical Colitis Activity Index; SD, standard deviation; TSAT, transferrin saturation; UC, ulcerative colitis.

\subsubsection{Efficacy Outcomes}

The impact of ferric maltol on hemoglobin and iron indices in the phase III trials in adults is summarized in Table 2.

The phase III AEGIS 1/2 IBD and CKD studies met their respective primary endpoints, with statistically significant differences between ferric maltol and the placebo in the change in hemoglobin from baseline to week $12(p<0.0001)$ [62] and week $16(p=0.01)$ [72], respectively. On average, hemoglobin increased by $\geq 2 \mathrm{~g} / \mathrm{dL}$ by week 12 in the AEGIS $1 / 2$ IBD study, which is deemed to be a clinically meaningful rise [62], and the level was sustained up to week 64 [64]. The increase in hemoglobin in the CKD study was smaller, reflecting the complex interaction between inflammation, kidney dysfunction, and iron regulation in these patients [72]. Nevertheless, the increase was significantly greater with ferric maltol than with the placebo $(p=0.01)$, and patients with CKD who were treated for up to 52 weeks achieved ongoing increases in hemoglobin over time [72]. In the phase III IBD head-to-head study, although ferric maltol did not meet the prespecified short-term noninferiority margin versus IV ferric carboxymaltose at the week 12 primary endpoint, the ferric maltol group did achieve a mean increase in hemoglobin of $>2 \mathrm{~g} / \mathrm{dL}$ at that timepoint, which was sustained for up to 52 weeks; at the end of the study, mean hemoglobin levels were similar in the oral and IV arms [71]. These data demonstrate that ferric maltol can provide effective long-term iron replacement and correction of anemia in patients with chronic underlying inflammatory conditions.

In subgroup analyses, ferric maltol had a consistent impact on hemoglobin concentrations regardless of the baseline hemoglobin level, according to a post hoc analysis of the phase IIIB IBD head-to-head study. In the ferric maltol arm, the mean hemoglobin increase from baseline to week 12 was $2.92 \mathrm{~g} / \mathrm{dL}$ in patients with baseline hemoglobin $<9.5 \mathrm{~g} / \mathrm{dL}$ (baseline mean $8.6 \mathrm{~g} / \mathrm{dL} ; n=38$ ) and $2.35 \mathrm{~g} / \mathrm{dL}$ in patients with baseline hemoglobin $\geq 9.5 \mathrm{~g} / \mathrm{dL}$ (baseline mean $10.6 \mathrm{~g} / \mathrm{dL} ; n=87$ ), and $70 \%$ versus $67 \%$, respectively, achieved $a \geq 2 \mathrm{~g} / \mathrm{dL}$ increase or normalization of hemoglobin [84]. The severity of the underlying IBD or CKD activity, as measured by clinical index scores or inflammatory markers such as C-reactive protein $[62,65,67]$, and the use of proton pump inhibitors in patients with IBD [63] did not affect the efficacy of ferric maltol. 
Table 2. Phase III studies of ferric maltol in adults: key efficacy outcomes.

\begin{tabular}{|c|c|c|c|c|c|c|}
\hline Study & Mean $\mathrm{Hb}$ and Change from Baseline & $\mathrm{Hb}$ Responder Rate ${ }^{1}$ & $\begin{array}{c}\text { Proportion of Patients } \\
\text { Achieving Hb Normalization }{ }^{2}\end{array}$ & $\begin{array}{c}\text { Proportion of Patients } \\
\text { Achieving } \geq 2 \mathrm{~g} / \mathrm{dL} \text { Increase in } \\
\mathrm{Hb}\end{array}$ & Mean Ferritin & Mean TSAT \\
\hline $\begin{array}{c}\text { Phase III IBD (AEGIS 1/2) } \\
{[62,64]}\end{array}$ & $\begin{array}{c}\text { Baseline } \\
\text { FM: } 11.0 \mathrm{~g} / \mathrm{dL} \\
\text { Placebo: } 11.1 \mathrm{~g} / \mathrm{dL} \\
\text { Week } 12 \\
\text { FM: } 13.2 \mathrm{~g} / \mathrm{dL} \\
\text { Placebo: } 11.2 \mathrm{~g} / \mathrm{dL} \\
\text { Mean (SE) difference in change from } \\
\text { baseline to week } 12 \\
\text { FM vs. placebo: } 2.25(0.12) \mathrm{g} / \mathrm{dL} \\
\text { p } 0.0001 \\
\text { Up to week } 64 \\
\text { DB FM to OL FM: } 13.95 \mathrm{~g} / \mathrm{dL} \\
\text { DB placebo to OL FM: } 13.33 \mathrm{~g} / \mathrm{dL}\end{array}$ & NR & $\begin{array}{c}\text { Week } 12 \\
\text { FM: 66\% Placebo: } 13 \% \\
\text { Up to week } 64 \\
\text { DB FM/placebo to OL FM: } 86 \%\end{array}$ & $\begin{array}{c}\text { Week } 12 \\
\text { FM: } 56 \% \text { Placebo: } 0 \\
\text { Up to week } 64 \% \text { NR }\end{array}$ & 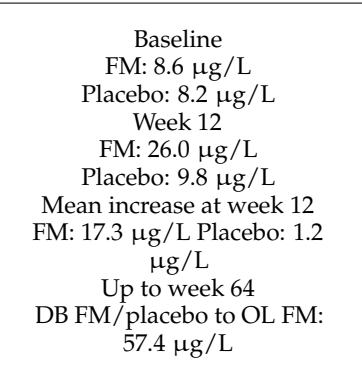 & $\begin{array}{c}\text { Baseline } \\
\text { FM: } 10.6 \% \\
\text { Placebo: } 9.5 \% \\
\text { Week } 12 \\
\text { FM: } 28.5 \% \\
\text { Placebo: } 9.8 \% \\
\text { Mean increase at week } 12 \\
\text { FM: } 18.0 \text { percentage points } \\
\text { Placebo: }-0.4 \text { percentage } \\
\text { points } \\
\text { Up to week } 64 \\
\text { DB FM } 6 \text { placebo to OL FM: } 29 \%\end{array}$ \\
\hline Phase IIIB IBD (H2H) [71] & $\begin{array}{c}\text { ITT population } \\
\text { Baseline } \\
\text { FM: } 10.0 \mathrm{~g} / \mathrm{dL} \\
\text { IV FCM: } 10.1 \mathrm{~g} / \mathrm{dL} \\
\text { Week } 12 \\
\text { FM: } 12.5 \mathrm{~g} / \mathrm{dL} \\
\text { IV FCM: } 13.2 \mathrm{~g} / \mathrm{dL} \\
\text { LSM difference }(95 \% \mathrm{CI}) \text { between } \\
\text { groups at week } 12 \\
\text { FM-IV FCM: }-0.6(-1.0 \text { to }-0.2) \mathrm{g} / \mathrm{dL} \\
p=0.002 \\
\text { Up to week } 52 / \mathrm{EoT} \\
\text { FM: } 12.8 \mathrm{~g} / \mathrm{dL} \\
\text { IV FCM: } 13.0 \mathrm{~g} / \mathrm{dL}\end{array}$ & $\begin{array}{c}\text { ITT population } \\
\text { Week } 12 \\
\text { FM: } 67 \% \text { IV FCM: } 84 \% \\
\text { Risk difference }(95 \% \text { CI) } \\
\text { FM-IV FCM: }-0.17(-0.28 \text { to } \\
-0.06) \\
\text { PP population } \\
\text { Week } 12 \\
\text { FM: } 68 \% \\
\text { IV FCM: } 85 \% \\
\text { Risk difference }(95 \% \text { CI }) \\
\text { FM-IV FCM: }-0.17(-0.30 \text { to } \\
0.05)^{3}\end{array}$ & $\begin{array}{l}\text { ITT population } \\
\text { Week } 12 \\
\text { FM: } 55 \% \\
\text { IV FCM: } 81 \% \\
\text { Up to week } 52 / \text { EoT } \\
\text { NR }\end{array}$ & $\begin{array}{c}\text { ITT population } \\
\text { Week } 12 \\
\text { FM: } 61 \% \text { IV FCM: } 77 \% \\
\text { Up to week } 52 / \text { EoT } \\
\text { NR }\end{array}$ & $\begin{array}{c}\text { ITT population } \\
\text { Baseline } \\
\text { FM: } 16.6 \mu \mathrm{g} / \mathrm{L} \\
\text { IV FCM: } 9.2 \mu \mathrm{g} / \mathrm{L} \\
\text { Week } 12 \\
\text { FM: } 25.7 \mu \mathrm{g} / \mathrm{L} \\
\text { IV FCM: } 139.2 \mu \mathrm{g} / \mathrm{L} \\
\text { LSM difference }(95 \% \mathrm{CI}) \\
\text { between groups at week } 12 \\
\text { FM-IV FCM: }-113.1(-145.9 \text { to } \\
-80.2) \mu \mathrm{g} / \mathrm{L} \\
p<0.001 \\
\text { Up to week } 52 / \text { EoT } \\
\text { FM: } 58.9 \mu \mathrm{g} / \mathrm{L} \\
\text { IV FCM: } 103.4 \mu \mathrm{L} / \mathrm{L}\end{array}$ & NR \\
\hline Phase III CKD [72] & $\begin{array}{c}\text { Baseline } \\
\text { FM: } 10.1 \mathrm{~g} / \mathrm{dL} \\
\text { Placebo: } 10.0 \mathrm{~g} / \mathrm{dL} \\
\text { FMM } 0.5 \mathrm{~g} / \mathrm{dL} \\
\text { LSM change from waseline to } 16 \\
\text { Placebo: }-0.0 \mathrm{~g} / \mathrm{dL} \\
\text { LSM (SE) difference between groups at } \\
\text { week } 16 \\
\text { FM-placebo: } 0.5(0.2) \mathrm{g} / \mathrm{dL} \\
p=0.01 \\
\text { Up to week } 52 / \mathrm{EoT} \\
\text { DB FM to OL FM: } 10.9 \mathrm{~g} / \mathrm{dL} \\
\text { DB placebo to OL FM: } 10.9 \mathrm{~g} / \mathrm{dL}\end{array}$ & NR & $\begin{array}{c}\text { Week } 16 \\
\text { FM: } 27 \% \\
\text { Placebo: } 13 \% \\
\text { Up to week } 52 / \text { EoT } \\
\text { NR }\end{array}$ & $\begin{array}{c}\text { Week } 16 \\
\text { FM: } 6 \% \text { Placebo: } 0 \\
\text { Up to week 52/EoT } \\
\text { NR }\end{array}$ & $\begin{array}{c}\text { Baseline } \\
\text { FM: } 97.0 \mu \mathrm{g} / \mathrm{L} \\
\text { Placebo: } 104.2 \mu \mathrm{g} / \mathrm{L} \\
\text { LSM change from baseline to } \\
\text { week } 16 \\
\text { FM: } 25.4 \mu \mathrm{g} / \mathrm{L} \\
\text { Placebo: }-7.2 \mu \mathrm{g} / \mathrm{L} \\
\text { LSM (SE) difference between } \\
\text { groups at week } 16 \\
\text { FM-placebo: } 32.7(9.4) \mu \mathrm{g} / \mathrm{L} \\
p<0.001 \\
\text { Up to week } 52 / \mathrm{EoT} \\
\text { DB FM to OL FM: } 142.5 \mu \mathrm{g} / \mathrm{L} \\
\text { DB placebo to OL FM: } \\
146.3 \mu \mathrm{g} / \mathrm{L}\end{array}$ & $\begin{array}{c}\text { Baseline } \\
\text { FM: } 15.7 \% \\
\text { Placebo: } 15.6 \% \\
\text { LSM change from boseline to } \\
\text { week } 16 \\
\text { FM: } 3.8 \text { percentage points } \\
\text { Placebo: }-0.9 \text { percentage } \\
\text { points } \\
\text { LSM (SE) difference between } \\
\text { groups at week 16 } \\
\text { FM-placebo: } 4.6(1.1) \\
\text { percentage points } \\
p<0.001 \\
\text { Up to week 52/EoT } \\
\text { DB FM to OL FM: } 23.5 \% \\
\text { DB placebo to OL FM: } 21.4 \%\end{array}$ \\
\hline
\end{tabular}


Table 2. Cont.

\begin{tabular}{|c|c|c|c|c|c|c|}
\hline Study & Mean $\mathrm{Hb}$ and Change from Baseline & Hb Responder Rate ${ }^{1}$ & $\begin{array}{c}\text { Proportion of Patients } \\
\text { Achieving Hb Normalization }{ }^{2}\end{array}$ & $\begin{array}{c}\text { Proportion of Patients } \\
\text { Achieving } \geq 2 \mathrm{~g} / \mathrm{dL} \text { Increase in } \\
\mathrm{Hb}\end{array}$ & Mean Ferritin & Mean TSAT \\
\hline Phase IIIB PH [68] & $\begin{array}{c}\text { Baseline } \\
\text { FM: } 10.7 \mathrm{~g} / \mathrm{dL} \\
\text { Week } 12 \\
\text { FM: } 13.6 \mathrm{~g} / \mathrm{dL} \\
12: \\
\text { Median increase from baseline to week } \\
2.9 \mathrm{~g} / \mathrm{dL} \\
p<0.001\end{array}$ & NR & NR & NR & $\begin{array}{c}\text { Baseline } \\
\text { FM: } 13.1 \mu \mathrm{g} / \mathrm{L} \\
\text { Week } 12 \\
\text { FM: } 36.6 \mu \mathrm{g} / \mathrm{L} \\
p<0.001\end{array}$ & $\begin{array}{c}\text { Baseline } \\
\text { FM: } 7.5 \% \\
\text { Week } 12 \\
\text { FM: } 31.7 \% \\
p<0.001\end{array}$ \\
\hline
\end{tabular}

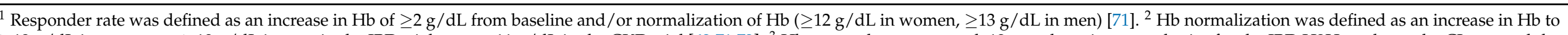

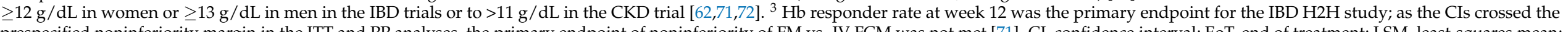

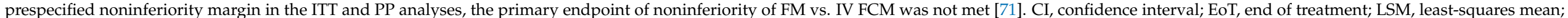

NR, not reported; SE, standard error. 
Measures of ferritin concentration and TSAT demonstrate that ferric maltol can increase iron availability by week 12 (IBD) and week 16 (CKD) [62,72]. As with hemoglobin, levels of these iron availability markers either increased or were maintained for up to a year with ongoing ferric maltol therapy in patients with underlying IBD or CKD [64,72]. In the IBD head-to-head study, IV ferric carboxymaltose therapy resulted in high ferritin levels at week 12, which had decreased somewhat by week 52; by contrast, levels in the ferric maltol arm increased substantially with ongoing treatment up to week 52, indicating steady replenishment of iron stores over time with this oral therapy [71]. In the phase I pediatric study, iron availability markers were increased at all ferric maltol doses even over the short study duration of 10 days [73].

In the phase IIIB study in patients with pulmonary hypertension $(n=22)$, the mean hemoglobin level increased significantly by $2.9 \mathrm{~g} / \mathrm{dL}$, from $10.7 \mathrm{~g} / \mathrm{dL}$ at baseline to $13.6 \mathrm{~g} / \mathrm{dL}$ after 12 weeks of treatment with ferric maltol $(p<0.001)$; ferritin and TSAT also increased significantly from baseline to week 12 (both $p<0.001$ ) [68]. In the UK real-world FRESH study $(n=59), 19$ patients achieved normalization of hemoglobin at week 12, and eight further patients achieved normalization after this timepoint; in addition, 16 patients achieved normalization of ferritin [69]. In the single-center, real-world study in London $(n=28)$, mean hemoglobin increased from $11.0 \mathrm{~g} / \mathrm{dL}$ at baseline to $12.2 \mathrm{~g} / \mathrm{dL}$ after a median of 16 weeks, while ferritin increased from $14 \mu \mathrm{g} / \mathrm{L}$ at baseline to $28 \mu \mathrm{g} / \mathrm{L}$ after a median of 16 weeks [70].

\subsubsection{Safety Findings}

In total, 492 adults and adolescents received ferric maltol in clinical studies [59,61,62,64,68,71-73], including 345 participants in trials planned to last 12 weeks (IBD and pulmonary hypertension) or 16 weeks (CKD); 293 of these 345 patients $(85 \%)$ completed 12 or 16 weeks of treatment $[62,68,71,72]$. In trials with a longer-term follow up, 331 patients received ferric maltol for more than 12 weeks (IBD) or 16 weeks (CKD), of whom $229(69 \%)$ completed treatment for up to 64 weeks (IBD) or 52 weeks (CKD) [64,71,72]. Among patients who stopped treatment before the end of the study, the most common reasons were adverse events (34/96 patients (35\%) who stopped ferric maltol prematurely vs. 14/53 patients (13\%) who stopped the placebo, and $2 / 19$ patients $(11 \%)$ who stopped IV ferric carboxymaltose prematurely) and physician or patient decision (28/96 (29\%), 18/53 (34\%), and $6 / 19(32 \%)$, respectively) [62,64,68,71,72].

In the phase III studies, the proportion of patients needing to stop ferric maltol therapy because of adverse events before week 12 or 16 was low $(\sim 10 \%)$ and similar rates in patients randomized to the placebo, even in the AEGIS 1/2 IBD study, which enrolled patients who had been unable to tolerate prior oral ferrous iron therapy [62]. The proportion increased slightly during the longer-term follow up in the AEGIS 1/2 IBD study, but it remained low in the IBD head-to-head and CKD studies [62,64,71,72]. In patients with IBD or CKD, the most common adverse events leading to discontinuation of ferric maltol treatment during long-term treatment (up to 52-64 weeks) were gastrointestinal, including abdominal pain in $2-3 \%$ (IBD only), constipation in $1-2 \%$, diarrhea in $1-3 \%$, and nausea in $1-2 \%$. For comparison, among patients with IBD given placebo, $3 \%$ stopped prematurely because of abdominal pain and $2 \%$ stopped because of diarrhea. Figure 1 summarizes the safety and tolerability of ferric maltol reported in the long-term phase III studies [62,64,68,71,72].

In the exploratory phase IIIB pulmonary hypertension study, treatment with ferric maltol was well tolerated by most patients, with only 2 of 22 patients $(9 \%)$ unable to complete 12 weeks of treatment (diarrhea $n=1$, pneumonia $n=1$ ) [68]. In the phase I pediatric study, 20 of the 37 adolescents (54\%) experienced an adverse event during the 10-day treatment period, with similar frequencies at each ferric maltol dose level. Only one adolescent discontinued treatment because of an adverse event (tonsilitis) [73]. 

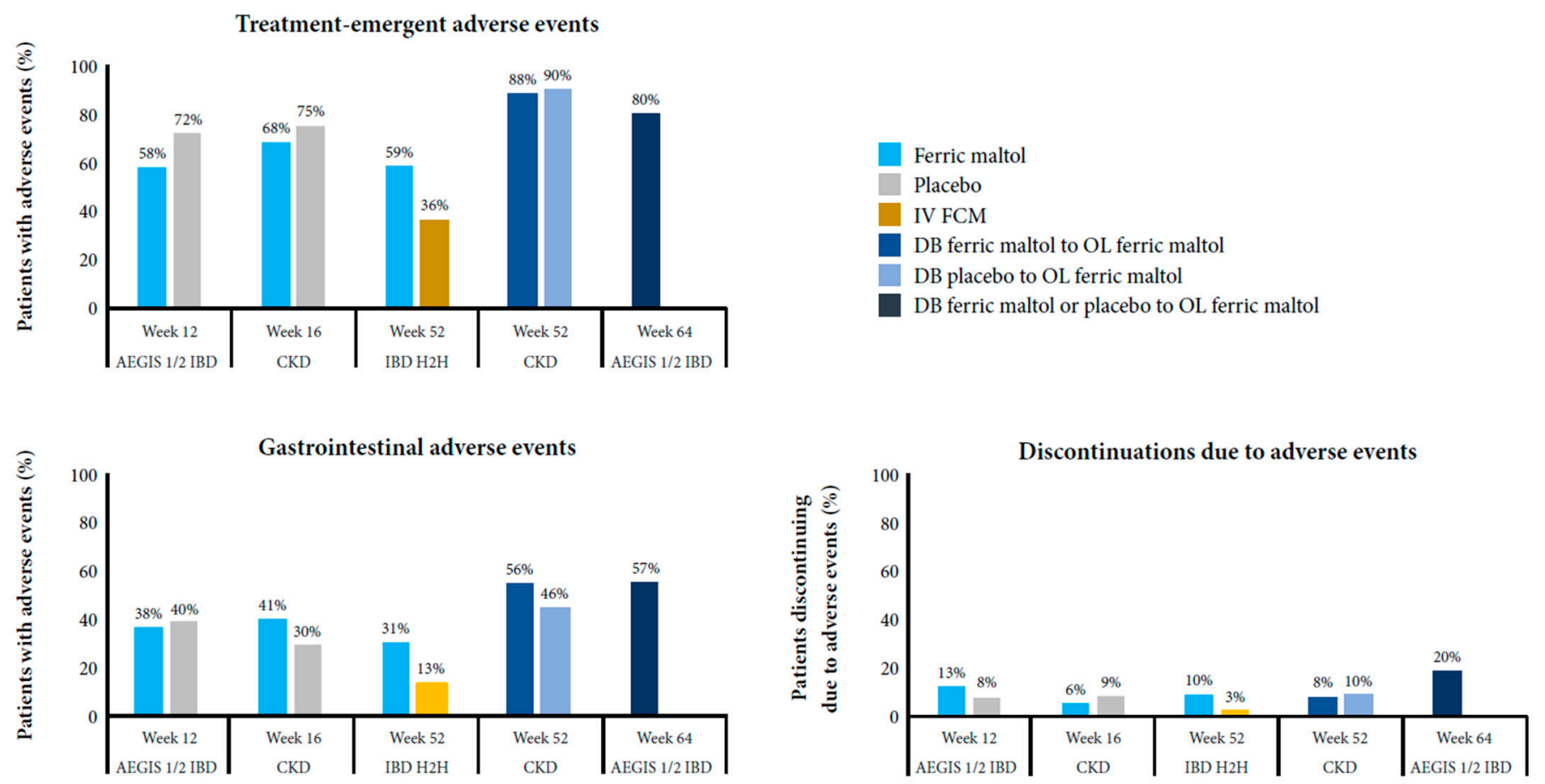

Figure 1. Safety and tolerability of ferric maltol in the long-term phase III studies $[62,64,71,72]$.

Overall, the most frequent treatment-emergent adverse events were gastrointestinal, which occurred at similar rates in patients treated with ferric maltol or placebo $(\sim 30-40 \%)$ up to week 12 or 16; the incidence increased slightly with longer treatment (46-57\%) in the AEGIS 1/2 IBD and CKD open-label extensions [62,64,71,72]. Gastrointestinal adverse events reported in all studies were constipation (4-6\% of patients with IBD, $13-16 \%$ of patients with CKD treated with ferric maltol for up to 52-64 weeks, 2-4\% of patients with IBD or CKD given placebo for $12-16$ weeks, and $<1 \%$ of patients with IBD treated with ferric carboxymaltose), diarrhea (5-14\%, 8-13\%, 9-10\%, and $<1 \%$, respectively), nausea (5\%, 12-13\%, 2-9\%, and 2\%, respectively), and vomiting ( $<1-4 \%, 0-8 \%, 3 \%$ (IBD only), and $3 \%$, respectively). In addition, nasopharyngitis was reported across all studies (8-18\%, $8-11 \%, 12 \%$ (IBD only), and 3\%, respectively). Gastrointestinal adverse events reported only in patients with IBD were abdominal pain (9-16\% of patients treated with ferric maltol over 52-64 weeks, $12 \%$ of patients given placebo for 12 weeks, and 3\% of patients treated with ferric carboxymaltose), flatulence (3-8\%, $0 \%$, and $0 \%$, respectively), and IBD flare (6-17\%, 12\%, and 7\%, respectively).

In the pulmonary hypertension study, $14 \%$ of patients had diarrhea [68]. Gastrointestinal events were also the most common adverse events in the 10-day pediatric study $(32 \%)$ [73].

Two reports of ferric maltol use in UK clinical practice support the favorable safety profile of ferric maltol in the real world. In the FRESH study, 19 of 59 patients with IBD (32\%) experienced adverse events (most commonly abdominal pain/discomfort [15\%]), and 30 of 59 patients (51\%) were treated for 12 weeks [69]. In the London study, 14 of 21 patients with IBD (67\%) tolerated ferric maltol for at least 1 month, including 5 of 10 patients (50\%) who had not previously tolerated oral ferrous iron therapy [70].

\section{Review of the Evidence and Clinical Implications}

To date, ferric maltol has been assessed in almost 750 adults and adolescents (661 in clinical trials and 87 in real-world studies) with a range of underlying conditions, including IBD, CKD, and pulmonary hypertension. The clinical trials were designed, as far as possible, to reflect the real-world settings in which ferric maltol will be used, with primary endpoints at 12 or 16 weeks followed by longer-term maintenance treatment in line with 
recommended treatment durations for oral iron therapies and current understanding of the time required for physiologic restoration of iron stores [45,88-91]. A further phase III study is being planned in infants and children (age range 1 month to 17 years) with IBD and irondeficiency anemia, which will use an oral suspension currently under investigation [92].

Underlying inflammatory conditions pose an ongoing risk of iron deficiency; thus, many patients require long-term iron replacement with a well-tolerated iron formulation. Ferric maltol fits this profile, with two-thirds of patients in open-label study arms staying on therapy for up to a year and thus benefitting from the long-term impact of ferric maltol on the body's iron needs $[64,71,72]$. Across all of the trial populations studied, ferric maltol has shown consistent and clinically relevant effects on hemoglobin and iron indices, with early increases in hemoglobin to meet erythropoiesis needs, followed by sustained replenishment of iron stores. In patients with IBD, 56-61\% achieved a $2 \mathrm{~g} / \mathrm{dL}$ increase in hemoglobin by week 12 . Although ferric maltol has not been directly compared with other oral iron replacement therapies, this is similar to rates reported with oral ferrous sulphate (58-71\% at weeks 8-12) [93], and a network meta-analysis indicated favorable hemoglobin improvements with ferric maltol (mean change $2.76 \mathrm{~g} / \mathrm{dL}$ versus placebo at 12 weeks) compared with other oral irons (mean change $1.04 \mathrm{~g} / \mathrm{dL}$ ) and IV irons (mean change 1.27-2.12 g/dL) after adjusting for baseline hemoglobin levels [66]. Over 52 weeks of treatment, ferric maltol provided similar hemoglobin increases to IV ferric carboxymaltose in patients with IBD, while ferritin levels increased substantially over time [71]. Nevertheless, IV iron should be preferred if a patient has severe anemia or more active IBD (compared with the disease activity in the clinical trials).

In chronic conditions such as iron-deficiency anemia requiring long-term therapy, it is important that treatment does not add to the overall burden of disease. Gastrointestinal adverse events have been widely reported with oral ferrous irons, including the most commonly used of these, ferrous sulphate. In a systematic review and meta-analysis, Tolkien et al. identified 43 trials involving 6831 adults, 3264 of whom received ferrous sulphate (range 7-226 patients/trial). Gastrointestinal adverse events were reported in $2-90 \%$ of patients (10-68\% in studies where $\geq 100$ patients received ferrous sulphate). The odds ratio for gastrointestinal adverse events in patients who received ferrous sulphate was 2.32 (95\% confidence interval 1.74-3.08, $p<0.0001)$ versus placebo and 3.05 (2.07-4.48, $p<0.001$ ) versus intravenous iron [30]. In the AEGIS 1/2 IBD study, the proportion of patients experiencing any adverse events up to week 12 was lower in the ferric maltol arm $(58 \%)$ than in the placebo arm $(72 \%)$, while the proportion of patients with gastrointestinal events was similar (38\% and 40\%, respectively) [62]. In the CKD study, the overall incidence of adverse events was again lower with ferric maltol $(68 \%)$ than with the placebo (75\%) up to week 16, although the incidence of gastrointestinal events was higher (40\% vs. 31\%) [72]. The incidence of any adverse events and of gastrointestinal events increased slightly with longer-term treatment (up to 52 or 64 weeks) in both studies [64,72]. In the IBD head-tohead study versus IV ferric carboxymaltose, $10 \%$ of patients in the ferric maltol arm stopped treatment because of adverse events compared with $3 \%$ in the IV arm. The frequency of gastrointestinal events was higher with ferric maltol (31\% up to week 52) than with IV iron $(13 \%)$, but the incidence in the ferric maltol arm was consistent with that reported with the placebo in the AEGIS 1/2 IBD study [62,71].

In a meta-analysis of IV versus oral ferrous irons, Bonovas et al. reported an odds ratio of $0.24(0.12-0.49)$ in favor of IV iron for treatment discontinuation due to adverse events, even though most of the oral iron studies may have had a selection bias toward favorable tolerability by excluding patients with known intolerance to previous oral irons [94], highlighting the need for a better tolerated oral iron formulation. In the AEGIS 1/2 IBD study, two-thirds of patients had previously stopped oral ferrous iron therapy because of adverse events; thus, they might be expected to have poor tolerance of a subsequent oral iron therapy, but in fact most patients were willing to continue ferric maltol for up to 64 weeks $[62,64]$. This finding is supported by the proof-of-concept study, in which 19 of 23 patients with known intolerance of prior oral therapies completed 3 months of 
ferric maltol therapy [61], and the reported real-world experience, in which $50 \%$ of patients, including a high proportion with prior oral therapy intolerance, were able to tolerate ferric maltol $[69,70]$.

Collectively, these findings indicate that prolonged treatment with ferric maltol provides sufficient iron to meet the body's erythropoietic needs, which could help reduce the need for erythropoiesis-stimulating agents or blood transfusions. At the same time, the amount of free iron in the gut is minimized, thereby reducing the risk of damage to the gut microbiome and of exacerbation of any underlying gastrointestinal disease.

\section{Summary and Future Directions}

The oral iron replacement therapy ferric maltol is licensed in Europe and the United States for the treatment of adults with iron deficiency with or without anemia. Of the 661 adults and adolescents who participated in the clinical trials, 492 received ferric maltol, and it has been widely used in clinical practice. Consistent evidence in a range of settings, including IBD, CKD, and pulmonary hypertension, indicates that ferric maltol is an effective and efficacious oral therapy for patients with iron deficiency and anemia, regardless of the underlying disease. The clinical impact of ferric maltol on iron deficiency without anemia or in other settings should be confirmed in future research. Future head-to-head studies of different oral iron replacement therapies in disease conditions where a direct comparison might be appropriate and ethically feasible especially in the context of drug safety could also help to guide clinical decision making. Nevertheless, with the exception of patients experiencing an IBD flare [88-90] and those requiring rapid iron replacement (best achieved with IV iron), we believe that ferric maltol is an appropriate treatment option for patients in whom long-term, convenient, and well-tolerated management of chronic iron deficiency is desired.

Supplementary Materials: The following are available online at https://www.mdpi.com/article/ $10.3390 / \mathrm{jcm} 10194448 / \mathrm{s} 1$, Figure S1. CONSORT flow diagram, Table S1. Designs of ferric maltol clinical trials.

Author Contributions: Conceptualization, all authors; methodology, all authors; writing—original draft preparation, all authors, with support from OPEN Health Communications; writing-review and editing, all authors. All authors have read and agreed to the published version of the manuscript.

Funding: The authors received no funding for this publication. Shield Therapeutics (UK) Ltd. (Gateshead, UK) paid for medical writing assistance.

Acknowledgments: Medical writing assistance in the development of this review was provided by Sara Black, ISMPP CMPP, of OPEN Health Communications (London, UK), with financial support from Shield Therapeutics (UK) Ltd. The authors retained full editorial control of the content and decision to publish.

Conflicts of Interest: C.S. has received consulting fees from AbbVie, Biogen, Ewopharma, Janssen, MSD, Pfizer, and Takeda; lecture fees and travel support from AbbVie, Berlin Chemie, Biogen, Ewopharma, Falk, Janssen, Merckle, med Update, MSD, Norgine, Novartis, Olympus, Pentax, Pfizer, Shire, Shield Therapeutics, and Takeda; and research support from AbbVie, Pentax, and Olympus. S.A. has served as the chair or member of data monitoring and safety boards for clinical trials of novel anti-malarials for MMV Medicines for Malaria Venture, Shin Poong Pharma, and Novartis, and for antiviral therapy for Ark Biosciences. N.K. has received consulting and speaker fees from Amgen, Otsuka, AstraZeneca, Aurinia, Vifor, and Opko. P.P. has participated in speaker bureaus for AstraZeneca and on advisory boards for Akebia, Ardelyx, Reata, Bayer, AstraZeneca, Tricida, and Gilead. Shield Therapeutics reviewed the manuscript for factual accuracy. 


\section{References}

1. World Health Organization. Vitamin and Mineral Nutrition Information System: Haemoglobin Concentrations for the Diagnosis of Anaemia and Assessment of Severity. Available online: https://www.who.int/vmnis/indicators/haemoglobin.pdf (accessed on 17 September 2021).

2. National Institute for Health and Care Excellence. Anaemia-Iron Deficiency. Available online: https://cks.nice.org.uk/topics/ anaemia-iron-deficiency / (accessed on 17 September 2021).

3. Gardner, W.; Kassebaum, N. Global, regional, and national prevalence of anemia and its causes in 204 countries and territories, 1990-2019. Curr. Dev. Nutr. 2020, 4, 830. [CrossRef]

4. Lopez, A.; Cacoub, P.; Macdougall, I.C.; Peyrin-Biroulet, L. Iron deficiency anaemia. Lancet 2016, 387, 907-916. [CrossRef]

5. Gargallo-Puyuelo, C.J.; Alfambra, E.; García-Erce, J.A.; Gomollon, F. Iron treatment may be difficult in inflammatory diseases: Inflammatory bowel disease as a paradigm. Nutrients 2018, 10, 1959. [CrossRef]

6. Abbaspour, N.; Hurrell, R.; Kelishadi, R. Review on iron and its importance for human health. J. Res. Med. Sci. 2014, 19, 164-174.

7. Agarwal, R. Nonhematological benefits of iron. Am. J. Nephrol. 2007, 27, 565-571. [CrossRef]

8. Gasche, C.; Lomer, M.C.; Cavill, I.; Weiss, G. Iron, anaemia, and inflammatory bowel diseases. Gut 2004, 53, 1190-1197. [CrossRef]

9. Kaitha, S.; Bashir, M.; Ali, T. Iron deficiency anemia in inflammatory bowel disease. World J. Gastrointest. Pathophysiol. 2015, 6, 62-72. [CrossRef] [PubMed]

10. Danese, S.; Hoffman, C.; Vel, S.; Greco, M.; Szabo, H.; Wilson, B.; Avedano, L. Anaemia from a patient perspective in inflammatory bowel disease: Results from the European Federation of Crohn's and Ulcerative Colitis Association's online survey. Eur. J. Gastroenterol. Hepatol. 2014, 26, 1385-1391. [CrossRef] [PubMed]

11. Ebner, N.; Jankowska, E.A.; Ponikowski, P.; Lainscak, M.; Elsner, S.; Sliziuk, V.; Steinbeck, L.; Kube, J.; Bekfani, T.; Scherbakov, N.; et al. The impact of iron deficiency and anaemia on exercise capacity and outcomes in patients with chronic heart failure-Results from the Studies Investigating Co-morbidities Aggravating Heart Failure. Int. J. Cardiol. 2016, $205,6-12$. [CrossRef] [PubMed]

12. Eriksson, D.; Goldsmith, D.; Teitsson, S.; Jackson, J.; van Nooten, F. Cross-sectional survey in CKD patients across Europe describing the association between quality of life and anaemia. BMC Nephrol. 2016, 17, 97. [CrossRef] [PubMed]

13. Nielsen, O.H.; Soendergaard, C.; Vikner, M.E.; Weiss, G. Rational management of iron-deficiency anaemia in inflammatory bowel disease. Nutrients 2018, 10, 82. [CrossRef]

14. González Alayón, C.; Pedrajas Crespo, C.; Marín Pedrosa, S.; Benítez, J.M.; Iglesias Flores, E.; Salgueiro Rodríguez, I.; Medina Medina, R.; García-Sánchez, V. Prevalence of iron deficiency without anaemia in inflammatory bowel disease and impact on health-related quality of life. Gastroenterol. Hepatol. 2018, 41, 22-29. [CrossRef]

15. Jankowska, E.A.; von Haehling, S.; Anker, S.D.; Macdougall, I.C.; Ponikowski, P. Iron deficiency and heart failure: Diagnostic dilemmas and therapeutic perspectives. Eur. Heart J. 2013, 34, 816-829. [CrossRef]

16. Halterman, J.S.; Kaczorowski, J.M.; Aligne, C.A.; Auinger, P.; Szilagyi, P.G. Iron deficiency and cognitive achievement among school-aged children and adolescents in the United States. Pediatrics 2001, 107, 1381-1386. [CrossRef] [PubMed]

17. Jáuregui-Lobera, I. Iron deficiency and cognitive functions. Neuropsychiatr. Dis. Treat. 2014, 10, 2087-2095. [CrossRef]

18. Covic, A.; Jackson, J.; Hadfield, A.; Pike, J.; Siriopol, D. Real-world impact of cardiovascular disease and anemia on quality of life and productivity in patients with non-dialysis-dependent chronic kidney disease. Adv. Ther. 2017, 34, 1662-1672. [CrossRef] [PubMed]

19. Beard, J.L. Iron biology in immune function, muscle metabolism and neuronal functioning. J. Nutr. 2001, 131, 568S-580S [CrossRef] [PubMed]

20. Andreini, C.; Putignano, V.; Rosato, A.; Banci, L. The human iron-proteome. Metallomics 2018, 10, 1223-1231. [CrossRef] [PubMed]

21. Chua, A.C.; Graham, R.M.; Trinder, D.; Olynyk, J.K. The regulation of cellular iron metabolism. Crit. Rev. Clin. Lab. Sci. 2007, 44, 413-459. [CrossRef]

22. Ganz, T.; Nemeth, E. Hepcidin and iron homeostasis. Biochim. Biophys. Acta 2012, 1823, 1434-1443. [CrossRef]

23. Silva, B.; Faustino, P. An overview of molecular basis of iron metabolism regulation and the associated pathologies. Biochim. Biophys. Acta 2015, 1852, 1347-1359. [CrossRef]

24. Dignass, A.U.; Gasche, C.; Bettenworth, D.; Birgegård, G.; Danese, S.; Gisbert, J.P.; Gomollon, F.; Iqbal, T.; Katsanos, K.; Koutroubakis, I.; et al. European consensus on the diagnosis and management of iron deficiency and anaemia in inflammatory bowel diseases. J. Crohn's Colitis 2015, 9, 211-222. [CrossRef]

25. Alleyne, M.; Horne, M.K.; Miller, J.L. Individualized treatment for iron-deficiency anemia in adults. Am. J. Med. 2008, 121, 943-948. [CrossRef]

26. Kumar, A.; Brookes, M.J. Iron therapy in inflammatory bowel disease. Nutrients 2020, 12, 3478. [CrossRef]

27. D'amico, F.; Peyrin-Biroulet, L.; Danese, S. Oral iron for IBD patients: Lessons learned at time of COVID-19 pandemic. J. Clin. Med. 2020, 9, 1536. [CrossRef] [PubMed]

28. Girelli, D.; Ugolini, S.; Busti, F.; Marchi, G.; Castagna, A. Modern iron replacement therapy: Clinical and pathophysiological insights. Int. J. Hematol. 2018, 107, 16-30. [CrossRef] [PubMed]

29. Lund, E.K.; Wharf, S.G.; Fairweather-Tait, S.J.; Johnson, I.T. Oral ferrous sulfate supplements increase the free radical-generating capacity of feces from healthy volunteers. Am. J. Clin. Nutr. 1999, 69, 250-255. [CrossRef] [PubMed] 
30. Tolkien, Z.; Stecher, L.; Mander, A.P.; Pereira, D.I.; Powell, J.J. Ferrous sulfate supplementation causes significant gastrointestinal side-effects in adults: A systematic review and meta-analysis. PLoS ONE 2015, 10, e0117383. [CrossRef]

31. Carrier, J.; Aghdassi, E.; Platt, I.; Cullen, J.; Allard, J.P. Effect of oral iron supplementation on oxidative stress and colonic inflammation in rats with induced colitis. Aliment. Pharmacol. Ther. 2001, 15, 1989-1999. [CrossRef] [PubMed]

32. Yilmaz, B.; Li, H. Gut microbiota and iron: The crucial actors in health and disease. Pharmaceuticals 2018, 11, 98. [CrossRef]

33. Eckstein, R.P.; Symons, P. Iron tablets cause histopathologically distinctive lesions in mucosal biopsies of the stomach and esophagus. Pathology 1996, 28, 142-145. [CrossRef]

34. Kaye, P.; Abdulla, K.; Wood, J.; James, P.; Foley, S.; Ragunath, K.; Atherton, J. Iron-induced mucosal pathology of the upper gastrointestinal tract: A common finding in patients on oral iron therapy. Histopathology 2008, 53, 311-317. [CrossRef] [PubMed]

35. Laine, L.A.; Bentley, E.; Chandrasoma, P. Effect of oral iron therapy on the upper gastrointestinal tract. A prospective evaluation. Dig. Dis. Sci. 1988, 33, 172-177. [CrossRef]

36. DeLoughery, T.G. Safety of oral and intravenous iron. Acta Haematol. 2019, 142, 8-12. [CrossRef] [PubMed]

37. Jaeggi, T.; Kortman, G.A.; Moretti, D.; Chassard, C.; Holding, P.; Dostal, A.; Boekhorst, J.; Timmerman, H.M.; Swinkels, D.W.; Tjalsma, H.; et al. Iron fortification adversely affects the gut microbiome, increases pathogen abundance and induces intestinal inflammation in Kenyan infants. Gut 2015, 64, 731-742. [CrossRef] [PubMed]

38. Paganini, D.; Zimmermann, M.B. The effects of iron fortification and supplementation on the gut microbiome and diarrhea in infants and children: A review. Am. J. Clin. Nutr. 2017, 106, 1688s-1693s. [CrossRef] [PubMed]

39. Parmanand, B.A.; Kellingray, L.; Le Gall, G.; Basit, A.W.; Fairweather-Tait, S.; Narbad, A. A decrease in iron availability to human gut microbiome reduces the growth of potentially pathogenic gut bacteria: An in vitro colonic fermentation study. J. Nutr. Biochem. 2019, 67, 20-27. [CrossRef]

40. World Health Organization. WHO Model List of Essential Medicines: Ferrous salt. Available online: https://list.essentialmeds. org/recommendations / 143 (accessed on 17 September 2021).

41. Litton, E.; Xiao, J.; Ho, K.M. Safety and efficacy of intravenous iron therapy in reducing requirement for allogeneic blood transfusion: Systematic review and meta-analysis of randomised clinical trials. BMJ 2013, 347, f4822. [CrossRef]

42. O'Lone, E.L.; Hodson, E.M.; Nistor, I.; Bolignano, D.; Webster, A.C.; Craig, J.C. Parenteral versus oral iron therapy for adults and children with chronic kidney disease. Cochrane Database Syst. Rev. 2019, 2, Cd007857. [CrossRef]

43. Del Vecchio, L.; Longhi, S.; Locatelli, F. Safety concerns about intravenous iron therapy in patients with chronic kidney disease. Clin. Kidney J. 2016, 9, 260-267. [CrossRef]

44. Ribeiro, S.; Belo, L.; Reis, F.; Santos-Silva, A. Iron therapy in chronic kidney disease: Recent changes, benefits and risks. Blood Rev. 2016, 30, 65-72. [CrossRef]

45. Snook, J.; Bhala, N.; Beales, I.L.P.; Cannings, D.; Kightley, C.; Logan, R.P.; Pritchard, D.M.; Sidhu, R.; Surgenor, S.; Thomas, W.; et al . British Society of Gastroenterology guidelines for the management of iron deficiency anaemia in adults. Gut 2021. (Epub ahead of print). [CrossRef] [PubMed]

46. Auerbach, M.; Adamson, J.W. How we diagnose and treat iron deficiency anemia. Am. J. Hematol. 2016, 91, 31-38. [CrossRef] [PubMed]

47. Bokemeyer, B. Addressing unmet needs in inflammatory bowel disease. Drug Discov. Today 2015, 20, 1037-1039. [CrossRef] [PubMed]

48. Kontoghiorghes, G.J.; Kolnagou, A.; Demetriou, T.; Neocleous, M.; Kontoghiorghe, C.N. New era in the treatment of iron deficiency anaemia using trimaltol iron and other lipophilic iron chelator complexes: Historical perspectives of discovery and future applications. Int. J. Mol. Sci. 2021, 22, 5546. [CrossRef]

49. Barrand, M.A.; Callingham, B.A.; Hider, R.C. Effects of the pyrones, maltol and ethyl maltol, on iron absorption from the rat small intestine. J. Pharm. Pharmacol. 1987, 39, 203-211. [CrossRef]

50. Levey, J.A.; Barrand, M.A.; Callingham, B.A.; Hider, R.C. Characteristics of iron(III) uptake by isolated fragments of rat small intestine in the presence of the hydroxypyrones, maltol and ethyl maltol. Biochem. Pharmacol. 1988, 37, 2051-2057. [CrossRef]

51. Barrand, M.A.; Hider, R.C.; Callingham, B.A. The importance of reductive mechanisms for intestinal uptake of iron from ferric maltol and ferric nitrilotriacetic acid (NTA). J. Pharm. Pharmacol. 1990, 42, 279-282. [CrossRef]

52. Singh, R.K.; Barrand, M.A. Lipid peroxidation effects of a novel iron compound, ferric maltol. A comparison with ferrous sulphate. J. Pharm. Pharmacol. 1990, 42, 276-279. [CrossRef]

53. Barrand, M.A.; Callingham, B.A. Evidence for regulatory control of iron uptake from ferric maltol across the small intestine of the rat. Br. J. Pharmacol. 1991, 102, 408-414. [CrossRef]

54. Barrand, M.A.; Callingham, B.A.; Dobbin, P.; Hider, R.C. Dissociation of a ferric maltol complex and its subsequent metabolism during absorption across the small intestine of the rat. Br. J. Pharmacol. 1991, 102, 723-729. [CrossRef]

55. Kelsey, S.; Hider, R.; Bloor, J.; Blake, D.; Gutteridge, C.; Newland, A. Absorption of low and therapeutic doses of ferric maltol, a novel ferric iron compound, in iron deficient subjects using a single dose iron absorption test. J. Clin. Pharm. Ther. 1991, 16, 117-122. [CrossRef]

56. Maxton, D.; Thompson, R.; Hider, R. Absorption of iron from ferric hydroxypyranone complexes. Br. J. Nutr. 1994, 71, 203-207. [CrossRef]

57. Reffitt, D.M.; Burden, T.J.; Seed, P.T.; Wood, J.; Thompson, R.P.; Powell, J.J. Assessment of iron absorption from ferric trimaltol. Ann. Clin. Biochem. 2000, 37, 457-466. [CrossRef] 
58. Amin, S.; Nisar, S.; Kazmi, S.A. Stopped-flow kinetic study of reduction of ferric maltol complex by ascorbate. J. Adv. Chem. 2016, 12, 4338-4341. [CrossRef]

59. Bokemeyer, B.; Krummenerl, A.; Maaser, C.; Howaldt, S.; Mroß, M.; Mallard, N. Randomized open-label phase 1 study of the pharmacokinetics of ferric maltol in inflammatory bowel disease patients with iron deficiency. Eur. J. Drug Metab. Pharmacokinet. 2017, 42, 229-238. [CrossRef] [PubMed]

60. Mahalhal, A.; Mansfield, J.; Sampson, M.; Lewis, S.; Lamb, C.; Frau, A.; Pritchard, D.; Probert, C. PTH-102 Ferric maltol, unlike ferrous sulphate, does not adversely affect the intestinal microbiome. Gut 2019, 68, A84. [CrossRef]

61. Harvey, R.; Reffitt, D.; Doig, L.; Meenan, J.; Ellis, R.; Thompson, R.; Powell, J. Ferric trimaltol corrects iron deficiency anaemia in patients intolerant of iron. Aliment. Pharmacol. Ther. 1998, 12, 845-848. [CrossRef]

62. Gasche, C.; Ahmad, T.; Tulassay, Z.; Baumgart, D.C.; Bokemeyer, B.; Büning, C.; Howaldt, S.; Stallmach, A. Ferric maltol is effective in correcting iron deficiency anemia in patients with inflammatory bowel disease: Results from a phase- 3 clinical trial program. Inflamm. Bowel Dis. 2015, 21, 579-588. [CrossRef] [PubMed]

63. Büning, C.; Ahmad, T.; Bokemeyer, B.; Elisei, W.; Picchio, M.; Penna, A.; Giorgetti, G. Correcting iron deficiency anaemia in IBD with oral ferric maltol: Use of proton pump inhibitors does not affect efficacy. J. Crohn's Colitis 2015, 9, S339-S340. [CrossRef]

64. Schmidt, C.; Ahmad, T.; Tulassay, Z.; Baumgart, D.; Bokemeyer, B.; Howaldt, S.; Stallmach, A.; Büning, C.; AEGIS Study Group. Ferric maltol therapy for iron deficiency anaemia in patients with inflammatory bowel disease: Long-term extension data from a Phase 3 study. Aliment. Pharmacol. Ther. 2016, 44, 259-270. [CrossRef] [PubMed]

65. Quraishi, N.; Smith, S.; Ward, D.; Sharma, N.; Sampson, M.; Tselepis, C.; Iqbal, T. PTH-113 Disease activity affects response to enteral iron supplementation: Post-hoc analysis of data from the AEGIS study. Gut 2017, 66, A262. [CrossRef]

66. Jones, T.; Forsythe, A.; Sampson, M.; Tremblay, G.; Dolph, M. PSY19—Using a Bayesian network meta-analysis (NMA) to compare ferric maltol to treatments for iron deficiency and iron deficiency anaemia excluding CHF and CKD patients. Value Health 2018, 21, S439. [CrossRef]

67. Kopyt, N.P. Effect of oral ferric maltol on iron parameters in patients with chronic kidney disease (CKD) and varying degrees of inflammation: A randomized, controlled trial. J. Am. Soc. Nephrol. 2018, 29, 176.

68. Olsson, K.M.; Fuge, J.; Brod, T.; Kamp, J.C.; Schmitto, J.; Kempf, T.; Bauersachs, J.; Hoeper, M.M. Oral iron supplementation with ferric maltol in patients with pulmonary hypertension. Eur. Respir. J. 2020, 56, 2000616. [CrossRef] [PubMed]

69. Cummings, J.F.; Fraser, A.; Stansfield, C.; Beales, I.; Sebastian, S.; Hoque, S. Ferric maltol Real-world Effectiveness Study in Hospital practice (FRESH): Clinical characteristics and outcomes of patients with inflammatory bowel disease receiving ferric maltol for iron-deficiency anaemia in the UK. BMJ Open Gastroenterol. 2021, 8, e000530. [CrossRef]

70. Oppong, P.; Lovato, S.; Akbar, A. PTU-124 Real world tolerability \& efficacy of oral ferric maltol (Feraccru) in IBD associated anaemia. Gut 2018, 67, A252-A253. [CrossRef]

71. Howaldt, S.; Domènech, E.; Martinez, N.; Schmidt, C.; Bokemeyer, B. Long-term effectiveness of oral ferric maltol versus intravenous ferric carboxymaltose for the treatment of iron-deficiency anemia in patients with inflammatory bowel disease: A randomized controlled noninferiority trial. Inflamm. Bowel Dis. 2021. (Epub ahead of print). [CrossRef]

72. Pergola, P.E.; Kopyt, N.P. Oral ferric maltol for the treatment of iron-deficiency anemia in patients with chronic kidney disease: Phase 3, multicenter, randomized, placebo-controlled trial and open-label extension. Am. J. Kidney Dis. 2021. (Epub ahead of print). [CrossRef]

73. Allen, S.; Auth, M.K.-H.; Kim, J.J.; Vadamalayan, B. Safety, tolerability and pharmacokinetics of oral ferric maltol in children with iron deficiency: Phase 1 study. JPGN Rep. 2021, 2, e090. [CrossRef]

74. Tremblay, G.; Dolph, M.; Jones, T.; Forsythe, A.; Mellor, L.; Sampson, M. PSY94-Cost-effectiveness model comparing therapeutic strategies for treating iron deficiency anaemia (IDA) in inflammatory bowel disease (IBD). Value Health 2018, 21, S452. [CrossRef]

75. Tremblay, G.; Forsythe, A.; Rabe, A.; Jones, T. Comparing short form-6 dimension (SF-6D) derived utilities and EuroQol questionnaire -5 dimensions (EQ-5D) mapped utilities among patients with iron deficiency in inflammatory bowel disease (ID-IBD) treated with ferric maltol (FM) vs placebo. Value Health 2018, 21, S86. [CrossRef]

76. Hjelmgren, J.; Ericson, O.; Niska, P.; Kukkonen, P.; Birgegård, G. PSY98-Cost-effectiveness analysis of ferric maltol versus ferric carboxymaltose for the treatment of iron deficiency anaemia in IBD patients in Sweden and Finland. Value Health 2018, 21, S452-S453. [CrossRef]

77. Lovato, S.; Oppong, P.; Akbar, A. Evaluation of ferric maltol as alternative to parenteral iron therapy in patients with inflammatory bowel disease (IBD) in terms of costs reductions and healthcare human resource utilisation. Hemasphere 2018, 2, 1066.

78. Schmidt, C.; Baumgart, D.; Bokemeyer, B.; Büning, C.; Howaldt, S.; Stallmach, A.; Singfield, C.; Tremblay, G.; Jones, T. P478 Treatment with ferric maltol associated with improvements in quality of life for IBD patients with iron deficiency anaemia. J. Crohn's Colitis 2018, 12, S346. [CrossRef]

79. Howaldt, S.; Jacob, I.; Sampson, M.; Akriche, F. P331 Productivity loss in patients with inflammatory bowel disease receiving treatment for iron deficiency anaemia: A comparison of ferric maltol and IV iron. J. Crohn's Colitis 2020, 14, S319-S320. [CrossRef]

80. Howaldt, S.; Jacob, I.; Sampson, M.; Akriche, F. P567 Impact of oral ferric maltol and IV iron on health-related quality of life in patients with iron deficiency anaemia and inflammatory bowel disease, and relationship with haemoglobin and serum iron. J. Crohn's Colitis 2020, 14, S478-S479. [CrossRef]

81. Howaldt, S.; Jacob, I.; Sampson, M.; Akriche, F. P685 Healthcare resource use associated with ferric maltol and IV iron treatment for iron deficiency anaemia in patients with inflammatory bowel disease. J. Crohn's Colitis 2020, 14, S558. [CrossRef] 
82. Howaldt, S.; Becker, C.K.; Becker, J.A.; Poinas, A.C. P592 Costs savings associated with ferric maltol and the reduced use of intravenous iron based on real world data. J. Crohn's Colitis 2021, 15, S541-S542. [CrossRef]

83. Mahalhal, A.; Frau, A.; Burkitt, M.D.; Ijaz, U.Z.; Lamb, C.A.; Mansfield, J.C.; Lewis, S.; Pritchard, D.M.; Probert, C.S. Oral ferric maltol does not adversely affect the intestinal microbiome of patients or mice, but ferrous sulphate does. Nutrients 2021, 13, 2269. [CrossRef]

84. Akriche, F.; Jacob, I.; Schmidt, C.; Howaldt, S. P420 Comparative efficacy and safety of oral ferric maltol in inflammatory bowel disease patients with mild-to-moderate vs. more severe iron deficiency anaemia. J. Crohn's Colitis 2021, 15, S424-S425. [CrossRef]

85. Orozco, M.N.; Arriaga, C.; Solomons, N.W.; Schümann, K. Equivalent effects on fecal reactive oxygen species generation with oral supplementation of three iron compounds: Ferrous sulfate, sodium iron EDTA and iron polymaltose. Ann. Nutr. Metab. 2012, 60, 108-114. [CrossRef] [PubMed]

86. Bertuccelli, G.; Marotta, F.; Zerbinati, N.; Cabeca, A.; He, F.; Jain, S.; Lorenzetti, A.; Yadav, H.; Milazzo, M.; Calabrese, F.; et al. Iron supplementation in young iron-deficient females causes gastrointestinal redox imbalance: Protective effect of a fermented nutraceutical. J. Biol. Regul. Homeost. Agents 2014, 28, 53-63. [PubMed]

87. European Medicines Agency. Feraccru: EPAR—Public Assessment Report. Available online: https://www.ema.europa.eu/en/ documents / assessment-report/ feraccru-epar-public-assessment-report_en.pdf (accessed on 17 September 2021).

88. European Medicines Agency. Feraccru (Ferric Maltol) Summary of Product Characteristics. Available online: https://www.ema. europa.eu/en/documents/product-information/feraccru-epar-product-information_en.pdf (accessed on 17 September 2021).

89. Food \& Drug Administration. Accrufer (Ferric Maltol) Prescribing Information. Available online: https://www.accessdata.fda. gov/drugsatfda_docs/label/2019/212320Orig1s0001bl.pdf (accessed on 17 September 2021).

90. Swissmedic. Feraccru Product Information. Available online: https:/ /www.swissmedicinfo.ch/?Feraccru (accessed on 17 September 2021).

91. British Columbia Guidelines and Protocols Advisory Committee. Iron Deficiency-Diagnosis and Management. Available online: https://www2.gov.bc.ca/assets/gov/health/practitioner-pro/bc-guidelines/iron-deficiency.pdf (accessed on 17 September 2021).

92. ClinicalTrials.gov. Four-Way Crossover Study to Compare Ferric Maltol Capsules and Oral Suspension in Healthy Volunteers. Available online: https:/ / www.clinicaltrials.gov/ct2/show / NCT04626414 (accessed on 17 September 2021).

93. Gordon, M.; Sinopoulou, V.; Iheozor-Ejiofor, Z.; Iqbal, T.; Allen, P.; Hoque, S.; Engineer, J.; Akobeng, A.K. Interventions for treating iron deficiency anaemia in inflammatory bowel disease. Cochrane Database Syst. Rev. 2021, 1, Cd013529. [CrossRef]

94. Bonovas, S.; Fiorino, G.; Allocca, M.; Lytras, T.; Tsantes, A.; Peyrin-Biroulet, L.; Danese, S. Intravenous versus oral iron for the treatment of anemia in inflammatory bowel disease: A systematic review and meta-analysis of randomized controlled trials. Medicine 2016, 95, e2308. [CrossRef] [PubMed] 